\title{
Long-tailed Visual Recognition via Gaussian Clouded Logit Adjustment
}

This paper was downloaded from TechRxiv (https://www.techrxiv.org).

LICENSE

CC BY 4.0

SUBMISSION DATE / POSTED DATE

$17-11-2021 / 19-11-2021$

CITATION

Li, Mengke; Cheung, Yiu-ming; Lu, Yang (2021): Long-tailed Visual Recognition via Gaussian Clouded Logit Adjustment. TechRxiv. Preprint. https://doi.org/10.36227/techrxiv.17031920.v1

$\mathrm{DOI}$

10.36227/techrxiv.17031920.v1 


\section{Long-tailed Visual Recognition via Gaussian Clouded Logit Adjustment}

\author{
Mengke Li Yiu-ming Cheung \\ Department of Computer Science, Hong Kong Baptist University \\ \{csmkli, ymc\}@comp.hkbu.edu.hk
}

\author{
Yang Lu \\ Xiamen University \\ luyang@xmu.edu.cn
}

\begin{abstract}
Long-tailed data is still a big challenge for deep neural networks, even though they have achieved great success on balanced data. We observe that vanilla training on longtailed data with cross-entropy loss makes the instance-rich head classes severely squeeze the spatial distribution of the tail classes, which leads to difficulty in classifying tail class samples. Furthermore, the original cross-entropy loss can only propagate gradient short-lively because the gradient in softmax form rapidly approaches zero as the logit difference increases. This phenomenon is called softmax saturation. It is unfavorable for training on balanced data, but can be utilized to adjust the validity of the samples in longtailed data, thereby solving the distorted embedding space of long-tailed problems. To this end, this paper therefore proposes the Gaussian clouded logit adjustment by Gaussian perturbing different class logits with varied amplitude. We define the amplitude of perturbation as cloud size and set relatively large cloud sizes to tail classes. The large cloud size can reduce the softmax saturation and thereby making tail class samples more active as well as enlarging the embedding space. To alleviate the bias in the classifier, we accordingly propose the class-based effective number sampling strategy with classifier re-training. Extensive experiments on benchmark datasets validate the superior performance of the proposed method.
\end{abstract}

\section{Introduction}

Deep neural networks (DNNs) have made a great success in a variety of visual recognition problems $[6,7,21,29]$ by virtue of the large-scale, high-quality, and annotated datasets. DNNs usually require the training dataset to be artificially balanced and have sufficient samples of each class. Unfortunately, from a practical perspective, object frequency usually follows a power law and typically exhibits a long-tailed distribution. Naive learning on such data is prone to undesirable bias towards the head classes which occupy the majority of the training samples [39]. Since tail classes have few training samples that cannot cover the

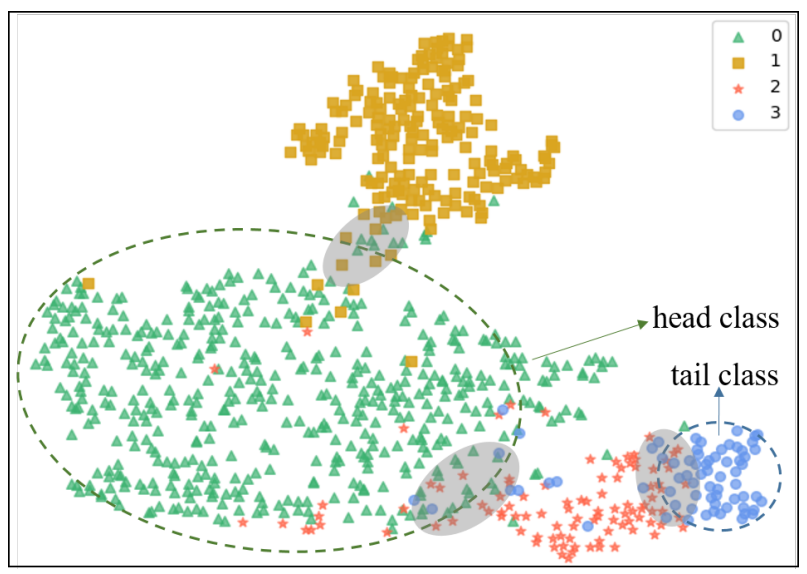

Figure 1. t-SNE visualization of the distorted embedding space. (Color for the best view.) The embeddings are calculated with ResNet-32 on a subset with four classes of CIFAR-10-LT. We randomly select four classes with the training numbers 500, 200, 100, and 50, respectively. The gray areas show the obscure regions between different classes.

real distribution in embedding space, their spatial span is severely compressed by head classes. In addition, a vast number of head class samples generate overwhelming discouraging gradients for tail classes. Thus, the classifier is biased towards the head classes. As a result, directly training on long-tailed data brings two key problems: 1) the distorted embedding space, and 2) the biased classifier.

In the literature, most of the recently proposed approaches only focus on addressing the second problem, i.e., the biased classifier. For example, Menon et al. [17] and Hong et al. [8] applied post-adjust strategy to the trained model to calibrate the class boundary. Nevertheless, the distorted embedding cannot be adjusted with the post-hoc calibration, which is not conducive to further improving the model performance. Most recently, the two-stage decoupling methods [2, 10, 32, 37, 42] have been proposed to obtain good embeddings in the first stage and then re-balance the classifier in the second stage. These methods obtain the representation by cross-entropy (CE) loss, which, however, leads to a severely uneven distributed embedding space. We 
implement a toy experiment to illustrate the distortion of the embedding space, as shown in Fig. 1. t-SNE [26] is utilized to visualize the features of a long-tailed subset from CIFAR-10 dataset. We can observe that the tail class occupies a much small spatial span than the head class. This is because the tail class with fewer samples cannot cover the ground truth distribution. Moreover, Fig. 1 also shows that there are obscure regions (i.e., the grey area) between different classes. Softmax saturation [3] is one of the factors of these obscure regions because it leads to insufficient training. These obscure regions have a severe effect on the tail classes but little on the head classes. Since tail class samples clustered around the class boundary aggravate their spatial squeezing, while the head class samples with enough variety can already cover the true distribution.

Softmax saturation refers to the inopportune early gradients vanishing produced by the softmax $[3,38]$, which weakens the validity of training samples and impedes model training. However, from another perspective, the seemingly harmful softmax saturation has the ability to balance the valid samples of different classes and thus help to calibrate the distortion of embedding space. Specifically, we disturb the logit of different classes with different amplitudes. We name the disturbed logit as Gaussian cloud logit (GCL) and the amplitude of the disturbance as cloud size, because we set the disturbance to a Gaussian distribution. The tail classes have few training samples and thus the training samples of them should be more valid. We therefore disturb the logit of tail classes with large relative cloud sizes to reduce the softmax saturation. In this way, tail class samples can provide more gradients without overfitting and thus indirectly affect their embedding space. In addition, A large cloud size of the tail class logit corresponds to the large cloud size on feature in the direction of the class anchor. Therefore, tail classes can have large margins towards the class boundary, so as to alleviate the severe uneven distribution between the head and tail classes. Conversely, the head classes are set to small cloud sizes, so that they can be automatically filtered out during training. Eventually, as shown in Fig. 2, the tail class samples can be pushed more away from the class boundary so as the distortion of the embedding space can be calibrated.

To address the biased classifier, we re-balance the training data with a class-wise sampling strategy. As training with GCL makes the validity of different classes varies, the so-called "effectiveness" [4] of them are different. Existing class-wise balanced sampling strategies will lead to excessive training of tail classes for GCL. We thereby propose the class-based effective number (CBEN) sampling strategy which is based on sample validity and label frequencies to re-balance the classifier. This simple but effective sampling strategy helps mitigate the classifier bias towards the head classes and further boost the performance of GCL.

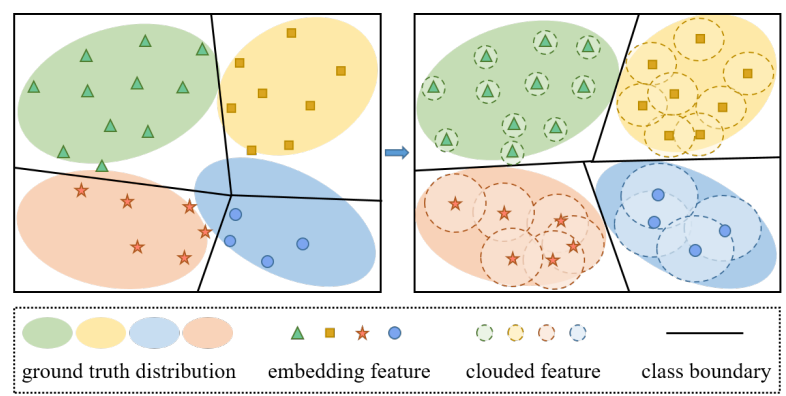

Figure 2. An overview of GCL. (Color for the best view.) The tail class logit is assigned to a larger sample cloud size than the head class, which corresponds to a large relative cloud size of the feature in the direction of the tail class anchor. In this way, the distortion of the embedding space can be calibrated well.

Extensive experiments on multiple commonly used longtailed recognition benchmark datasets demonstrate that the proposed GCL surpasses the recently proposed counterparts. In summary, the key contributions of our work are three-fold:

- We propose Gaussian clouded logit (GCL) adjustment method, which utilizes softmax saturation to balance the validity of different classes. An evenly distributed embedding can be obtained with the proposed GCL.

- We propose a simple but effective class-based effective number (CBEN) sampling strategy for re-balancing the classifier to avoid repeat training of tail classes. This sampling strategy can further boost the performance of GCL.

- Extensive experiments on popular long-tailed datasets demonstrate that the proposed method outperforms the state-of-the-art counterparts.

\section{Related Works}

Long-tailed classification is one of the long-standing research problems in machine learning. There are several kinds of approaches proposed to address it. This section briefly introduces the most related three regimes, namely, loss modification, logit adjustment, and decoupling representation.

\subsection{Loss Modification}

Modifying the loss function through re-weighting is the most intuitive method. Sample-wise re-weighting methods $[15,20]$ attempt to make the model pay more attention to the difficult samples by introducing fine-gained coefficients in the loss for imbalanced learning. For example, focal loss [15] introduces a tunable focusing parameter, which is negatively correlated with the predicted probability of the target class. This focusing parameter helps the 
model focus training on hard samples and prevents the numerous easy negatives from overwhelming. Class-wise reweighting methods $[4,9,11,23]$ assign the standard CE loss with category-specific parameters that are inversely proportional to the class frequencies. For example, Tan et al. [23] proposed equalization Loss, which utilizes a weight term to randomly ignore the discouraging gradients of head class samples. These methods can alleviate the data imbalance to a certain extent. However, the classification difficulty of a sample is not directly related to its corresponding class size. And another side effect of assigning higher weights to difficult samples/tail classes is overly focusing on harmful samples (e.g., noisy data or mislabeled data) [13].

\subsection{Logit Adjustment}

Logit adjustment assigns relatively large margins for tail classes. Most recently, Menon et al. [17] have proposed a logit adjustment (LA) method which is consistent for minimizing the balanced error. The logit shifting in LA of different classes is based on label frequencies of training data. Differently, LADE [8] calibrates the logit to the test set using the label distribution of test data, so that the test set can also be imbalanced. Tang et al. [24] adopted causal intervention to remove the "bad" SGD momentum and keep the "good" one to avoid the harmful causal effect for tail prediction. DisAlign [37] adjusts the logit by calibrating the prediction distribution to a balanced distribution. These methods can well adjust the classifier but without considering calibrating the embedding space. Another type of approach $[1,2]$ addresses long-tailed data by leaving large relative margins for tail classes during training. For example, label-distribution-aware margin (LDAM) loss proposed by Cao et al. [2] utilized Rademacher complexity to theoretically prove that the margin should be inversely proportional to a quarter power of label frequencies. The hard margin on target logit helps make the intro-class samples more compact, but does not truly enlarge the tail class span in embedding space.

\subsection{Decoupling Representation}

Many recent works have focused on improving the long-tailed visual recognition performance by decoupling the representation and classifier. Most recently, LDAMDRW [2] was proposed, which learns features in the first stage and adopts the deferred re-weighting (DRW) to finetune the decision boundary in the second stage. It significantly improves the long-tailed prediction accuracy, but the theoretical explanation of DRW is not clear. After that, Kang et al. [10] precisely pointed out that the learning process of representation and classifier can be decoupled into two separate stages. The representation learning is conducted on the original long-tailed data in the first stage and the classifier learning is performed on class-balanced re-sampling data in the second stage. A lot of works $[32,34,37,41]$ have further refined this strategy. For example, Zhang et al. [37] proposed an adaptive calibration function to calibrate the predicted logits of different classes into a balanced class prior in the second stage. Zhong et al. [41] proposed label distribution-based soft label to deal with different degrees of over-confidence for classes and can improve the classifier learning in the second stage. Another alternative direction is proposed by Zhou et al. [42], which splits the network structure into two branches that focus on learning the representation of head and tail classes, respectively. This method incorporates feature mixup [28] into a cumulative learning strategy and also achieved state-of-theart results. Following [42], Wang et al. [31] introduced contrastive learning into this bilateral-branch network, which further improved the long-tailed classification performance.

\section{Proposed Approach: Gaussian Clouded Logit (GCL)}

The key idea of our proposed GCL is to utilize the softmax saturation to automatically balance the valid samples of head and tail classes. The theoretical motivation and the formulation of the loss function of the proposed approach are presented as follows.

\subsection{Motivation}

Fig. 1 shows that the obscure region among different classes, especially the tail class is large. On important factor of this obscure region is the softmax saturation in CE loss [3]. Suppose $\{x, y\} \in \mathcal{T}$ represent a sample $\{x, y\}$ from the training set $\mathcal{T}$ with totally $N$ samples in $C$ classes, and $y \in\{1, \ldots, C\}$ is the ground truth label. The softmax loss function for the input image $x$ can be written as:

$$
\mathcal{L}(x)=-\log p_{y}, \text { with } p_{y}=\frac{e^{z_{y}}}{\sum_{j=1}^{C} e^{z_{j}}},
$$

where $z_{j}$ represents the predicted logit of class $j$. We use the subscript $y$ to represent the target class, namely, $z_{y}$ indicates the target logit and $z_{j}, j \neq y$ is the non-target logit.

In backward propagation, the gradients on $z_{i}$ is calculated by:

$$
\frac{\partial \mathcal{L}}{\partial z_{i}}= \begin{cases}p_{i}-1, & i=y \\ p_{i}, & i \neq y\end{cases}
$$

Without loss of generality, we use the binary classification to illustrate. Suppose $x$ is from class 1 , then, the gradients on $z_{1}$ is calculated by:

$$
\frac{\partial \mathcal{L}}{\partial z_{1}}=-\frac{1}{1+e^{z_{1}-z_{2}}}
$$

Fig. 3 shows that the gradient of the target class rapidly approaches zero with the increase of the target predicted 


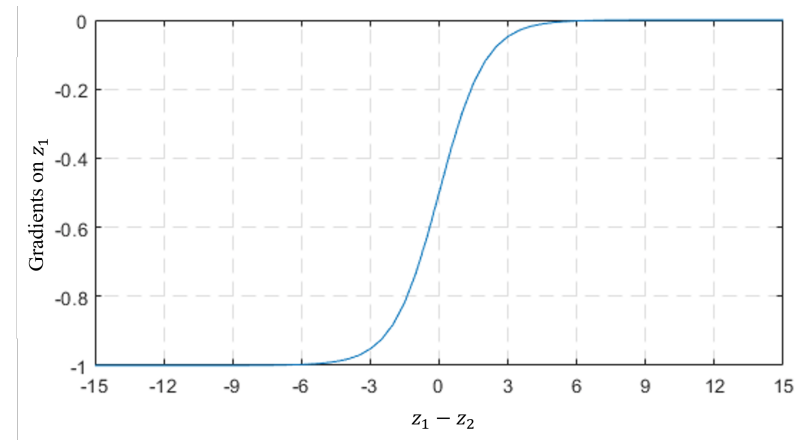

Figure 3 . The gradient on $z_{y}(y=1)$ in binary classification case. As the logit difference increases, the gradient rapidly approaches zero.

logit. Softmax can only slightly separate various classes, and lacks the power to evenly distribute each class in the embedded space. Therefore, there are many overlapping areas among each class. Especially under the circumstances of long-tailed classification, the tail classes features are not enough to cover the real distribution in embedding space. The early gradient vanish caused by soft saturation exacerbates the squeezing of their embedded space. A straightforward approach is to introduce hard margin [2, 5, 38]. However, the hard margin will cause the samples to shrink toward the class anchor and easy to overfit tail classes, which cannot evenly distribute the embedding space well. Fortunately, softmax saturation can help filter out the head class samples and make the tail class samples full participate in training. In this way, the tail classes can be pushed away from the head classes and indirectly enlarge their embedding space.

\subsection{Embedding Space Calibration}

Assuming that the features of different class samples satisfy Gaussian distribution. We can obtain a disturbed feature $\mathbf{f}^{c l d}$ of the input by Gaussian sampling, which is represented as:

$$
\mathbf{f}^{c l d} \triangleq \mathbf{f}+\delta \mathbf{E},
$$

where $f \in \mathbb{R}^{D}$ is the feature obtained from the embedding layer with the dimension of $D . \mathbf{E} \sim \mathcal{N}(\mathbf{u}, \boldsymbol{\Sigma})$ is the disturbance sampled from Gaussian distribution, and the mean vector and covariance matrix are represented by $\mathbf{u} \in \mathbb{R}^{D}$ and $\boldsymbol{\Sigma} \in \mathbb{R}^{D \times D}$, respectively. $\delta>0$ is a parameter that is used to adjust the amplitude of disturbance. In addition, $\delta$ should be a small number because a large disturbance will mislead the model. This disturbed feature is the input of the classifier. We use $\mathbf{W}=\left\{\mathbf{w}_{1}, \mathbf{w}_{2}, \cdots, \mathbf{w}_{C}\right\} \in \mathbb{R}^{D \times C}$ to represent the weight matrix of the classifier, where $\mathbf{w}_{j}$ represents the anchor vector of class $j$ in the classifier. Then, the corresponding disturbed logit $z_{j}^{c l d}$ of class $j$ is calcu- lated by:

$$
\begin{aligned}
z_{j}^{c l d} & =\mathbf{w}_{j}^{T} \mathbf{f}^{c l d}+\mathbf{b}_{j} \\
& =\mathbf{w}_{j}^{T} \mathbf{f}+\mathbf{b}_{j}+\mathbf{w}_{j}^{T}(\delta \mathbf{E}), \\
& =z_{j}+\delta\left(\mathbf{w}_{j}^{T} \mathbf{E}\right)
\end{aligned}
$$

As the range of $z_{j}^{c l d}$ is enlarged with random Gaussian disturbances, we call it Gaussian clouded logit, and $\delta\left(\mathbf{w}_{j}^{T} \mathbf{E}\right)$ is the clouded term. It needs to be noted that the clouded term has different degrees of influence on the final predicted results based on different predicted logits. It has a relatively small impact on $z_{j}^{c l d}$ when the original logit is large $z_{j}$. On the contrary, it will play a key role for $z_{j}^{c l d}$ when $z_{j}$ is small. As a result, we need to normalize the effect caused by different predicted logits and maintain the consistency of the influence of the clouded term. Inspired by [5, 29, 30], we normalize the clouded logits based on cosine distance. In this way, the norm of the embedding and the class anchor can be normalized to fix numbers. We use $s_{1}$ and $s_{2}$ to represent these two numbers. We name the normalized clouded logit as clouded cosine logit, which is calculated by:

$$
\begin{aligned}
\tilde{z}_{j}^{c l d} & =\frac{s_{1} \mathbf{w}_{j}^{T} \cdot s_{s} \mathbf{f}^{\text {cld }}}{\left\|\mathbf{w}_{j}^{T}\right\|\left\|\mathbf{f}^{c l d}\right\|} \\
& =s \cdot\left(\frac{\mathbf{w}_{j}^{T} \mathbf{f}}{\left\|\mathbf{w}_{j}^{T}\right\|\|\mathbf{f}+\delta \mathbf{E}\|}+\delta \frac{\mathbf{w}_{j}^{T} \mathbf{E}}{\left\|\mathbf{w}_{j}^{T}\right\|\|\mathbf{f}+\delta \mathbf{E}\|}\right),
\end{aligned}
$$

where $s=s_{1} \cdot s_{2}$ is a constant. In the first term of Eq. (6), $\|\mathbf{f}+\delta \mathbf{E}\| \approx\|\mathbf{f}\|$ because $\delta$ is a small number. In the second term, the norm of feature is normalized to $s_{1}$. Thus, $\tilde{z}_{j}^{\text {cld }}$ can be simplified as:

$$
\tilde{z}_{j}^{c l d} \approx s \cdot\left(\frac{\mathbf{w}_{j}^{T} \mathbf{f}}{\left\|\mathbf{w}_{j}^{T}\right\|\|\mathbf{f}\|}+\frac{\delta}{s_{1}} I_{j} \mathbf{E}\right),
$$

where $I_{j}$ is the identity vector that has the same direction as $\mathbf{w}_{j}^{T}$. In order to simplify the calculation, we make the clouded predicted cosine logit still satisfy the Gaussian distribution. Thus, we can set $\boldsymbol{\Sigma}=\sigma \mathbf{I}$, where $I \in \mathcal{R}^{D \times D}$ and $I$ is the identity matrix. Then, $z_{j}$ can be calculated by:

$$
\begin{aligned}
\tilde{z}_{j}^{c l d} & =s \cdot\left(\tilde{z}_{j}+\frac{\delta}{s_{1}} \varepsilon_{j}\right) \\
& \Leftrightarrow s \cdot\left(\tilde{z}_{j}+\delta_{j} \varepsilon\right)
\end{aligned}
$$

where $\tilde{z}_{j}=\cos \theta_{j}$ is the cosine distance between $\mathbf{f}$ and $\mathbf{w}_{j}$, and we name it as cosine logit. $\delta_{j}$ is the logit cloud size that depends on label frequencies.

To achieve the two goals mentioned in Sec. 3.1, i.e., 1) encourage tail class samples to participate more in training; 2) enlarge the embedding space for the tail classes, the size of logit cloud should be negatively correlated with the number of training samples. For the most frequent class, the diversity of training samples is sufficient and we set its 
logit cloud size to zero. While for tail classes, we use larger cloud sizes. This large relative cloud size of tail classes has three-fold advantages: 1) reduce the softmax saturation and thereby increase the training degree of tail classes; 2) different values are sampled randomly from the Gaussian cloud so as to avoid overfitting; 3 ) enlarge the margin of class boundary for tail classes and thus can calibrate the distortion of the embedding space. We therefore empirically set the cloud size for class $j$ as:

$$
\delta_{j}=\log n_{\max }-\log n_{j},
$$

where $n_{\max }$ is the sample numbers of the most frequent class. We experimentally verify the effectiveness of this cloud size adjustment strategy in Sec. 4.5.2 .

The Gaussian clouded logit difference $\Delta_{y_{-j}}$ between the target and non-target classes is:

$$
\begin{aligned}
\Delta_{y_{-} j} & =z_{y}^{c l d}-z_{j}^{c l d} \\
& =z_{y}-z_{j}+\varepsilon\left(\delta_{y}-\delta_{j}\right) .
\end{aligned}
$$

If $\varepsilon>0, \Delta_{y_{-} j}$ for tail classes will be increased. However, our goal is to reduce the logit difference to alleviate the softmax saturation for tail classes. In addition, a reduced logit corresponds to the feature that is relatively far from the class anchor. If the relatively distant feature can be predicted correctly, the closer one will be able to assign the right label. Therefore, we require $\varepsilon$ to be negative, and thus the clouded cosine logit can be written in the following form:

$$
\tilde{z}_{j}^{c l d}=s \cdot\left(\tilde{z}_{j}-\delta_{j}\|\varepsilon\|\right) .
$$

Take the clouded cosine logit into the original softmax, we can obtain the loss function of GCL:

$$
\mathcal{L}_{G C L}=-\frac{1}{N} \sum_{i} \log \frac{e^{\tilde{z}_{y_{i}}^{c l d}}}{\sum_{j} e^{\tilde{z}_{j}^{c l d}}} .
$$

\subsection{Classifier Re-balance}

The gradients derived in Eq. (2) demonstrate that the sample of the target class $y$ punishes the classifier weights $\mathbf{w}_{j}$ of non-target class $j, j \neq y$ w.r.t. $p_{j}$. The head classes have enormously greater training instances than tail classes. Therefore, the classifier weights of tail classes receive much more penalty than positive signals during training. Consequently, the classifier will bias towards the head classes, and the predicted logits of the tail classes will be seriously suppressed, resulting in low classification accuracy of the tail classes. A straightforward approach is to use the resampled data to re-train the classifier. We apply the classifier re-training (cRT) which was adopted by Kang et al. [10] and Wang et al. [33]. As the GCL loss enables different class samples to participate in training to different degrees, the effectiveness of different class samples is varied. Class-balanced sampling will lead to repeat training for

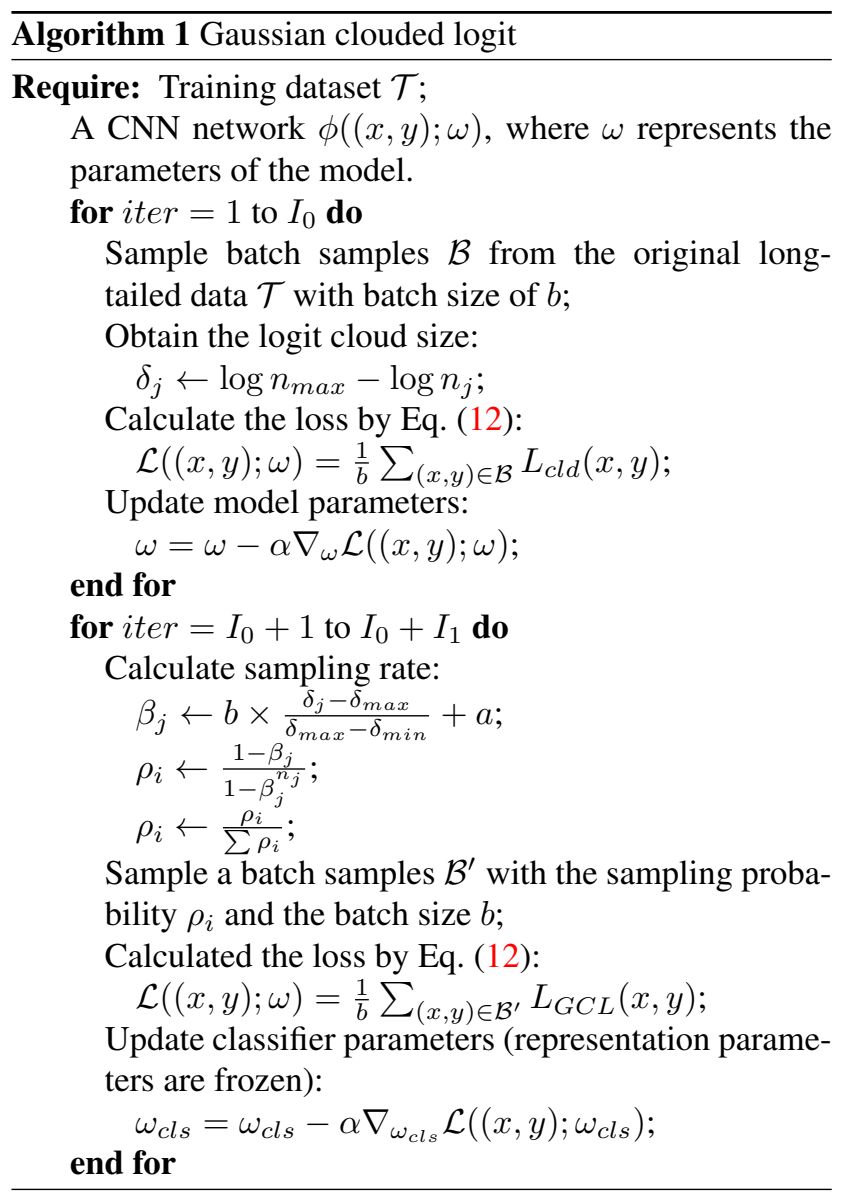

tail classes. Drawing on the effective number proposed by Cui et al. [4], we propose the class-based effective number (CBEN) sampling to avoid excessive training of tail classes. The sampling probability $\rho_{i}$ of for a sample from class $j$ is calculated by:

$$
\rho_{i}=\frac{1-\beta_{j}}{1-\beta_{j}^{n_{j}}}
$$

Since the sum of the sampling probability for all data need to be 1 , we need to normalize $\rho_{i}$ by $\rho_{i} \leftarrow \frac{\rho_{i}}{\sum \rho_{i}}$. $\beta_{j}$ reflects the validity of different class samples. The class samples with large cloud size participate more in training. Therefore, $\beta_{j}$ is positively correlated with cloud size $\delta_{j}$. We set $\beta_{j}$ as:

$$
\beta_{j}=b \times \frac{\delta_{j}-\delta_{\min }}{\delta_{\max }-\delta_{\min }}+a,
$$

so that $\beta_{j}$ can be in the region $[a, a+b]$, where $a$ and $b$ are the range hyper-parameters.

The overall training procedure of the proposed method is summarized in Algorithm 1. 


\section{Experiments}

\subsection{Datasets}

We use five benchmarks: long-tailed CIFAR datasets that include CIFAR-10-LT and CIFAR-100-LT, long-tailed ImageNet-2012 (ImageNet-LT), iNaturalist 2018 and longtailed Places-2 (Places-LT). The original version of CIFAR10/100 [14], ImageNet-2012 [22] and Places-2 [43] are all balanced datasets. We follow Cao et al. [4] and Cui et al. [2] to create long-tailed versions of CIFAR-10/100 and use the long-tailed versions of ImageNet-2012 and Places-2 produced by Liu et al. [16].

CIFAR-10/100-LT. The original CIFAR-10 and CIFAR100 consist of 10 and 100 classes, respectively. They both have 60,000 color images of size $32 \times 32$. 50,000 of them are used for training and the rest 10,000 images are for testing. Following [2, 4], we down-sampling training samples per class with the exponential function $n_{i}=n_{o_{i}} \times \mu^{i}$, where $i$ is the class index ( 0 -indexed), $n_{o_{i}}$ is the number of training samples in original CIFAR and $\mu \in(0,1)$. The test sets are kept unchanged. The imbalance ratio $\gamma$ is defined as the ratio of the sample size of the most and the least frequent classes, i.e. $\gamma=\max \left(n_{i}\right) / \min \left(n_{i}\right), i=1,2, \ldots, N$. We select the most widely used $\gamma=50,100$ and 200 in our experiments.

ImageNet-LT and Places-LT. The balanced versions of ImageNet-2012 and Places-2 are large-scale real-world datasets for classification and localization. We follow Liu et al.'s work [16] to construct the long-tailed version of these two datasets by truncating a subset with the Pareto distribution with the power value $\alpha=6$ from the balanced versions. The original balanced validation sets are used for testing. Overall, ImageNet-LT has $115.8 \mathrm{~K}$ training images from 1,000 categories with $\gamma=1,280 / 5$. Places-LT contains $62.5 \mathrm{~K}$ training images from 365 categories with $\gamma=4,980 / 5$.

iNaturalist 2018. iNaturalist 2018 [27] is a real-world fine-grained dataset for classification and detection, which naturally exhibits extremely imbalanced distribution. It contains $437.5 \mathrm{~K}$ training images and $24.4 \mathrm{~K}$ validation images from 8,142 categories. The official validation set is utilized as the test set in the experiments.

\subsection{Experimental Setting}

The parameters that need to be pre-set in the first stage are the Gaussian distribution parameters $\mu, \sigma^{2}$ and the region $[a, b]$ of sample validity $\beta_{j}$. We know that $\tilde{z}_{i} \in[-1,1]$, thus the maximum feature cloud size cannot exceed 1 . Because Gaussian distribution has a probability of $99.7 \%$ falling in $[\mu-3 \sigma, \mu+3 \sigma]$, we set $\mu=0$ and $\sigma=\frac{1}{3}$. We further clamp the $\varepsilon$ to $[-1,1]$ to prevent its amplitude from exceeding 1 . We set $\beta_{j} \in[0.999,0.9999]$, namely, $a=0.999$ and $b=0.0009$. Moreover, we normalize $\delta_{i}, i=\{1,2, \cdots, C\}$ by $\delta_{i} \triangleq \delta_{i} / \delta_{\max }$ to ensure that maximum value of $\delta_{i}$ does not exceed 1 . Similar with Zhong et al. [41], the mixup [35] strategy is also adopted in our experiment.

We use PyTorch [19] to implement all the backbones. SGD optimizer with momentum of 0.9 and the multi-step learning rate schedule are adopted. All the models are trained from scratch except ResNet-152 that is pre-trained on the original balanced version of ImageNet-2012. For the first stage, we select ResNet-32 as the backbone network and follow the setting in Cao et al. [2] for CIFAR-10/100LT. For the large-scale dataset, namely, ImageNet-LT, iNatralist 2018, and Places-LT, we mainly follow Kang et al. [10] except the learning rate schedule. For the second stage, i.e., re-balancing the classifier, we follow Kang et al. [10] for all datasets.

\subsection{Competing Methods}

To verify the effectiveness of the proposed method, we conduct extensive experiments to compare with previous methods including the following two groups:

Baseline Methods. We implement vanilla training with cross-entropy (CE) loss as one of our baseline methods. Much visual recognition works $[12,18,36,40]$ have shown the efficacy of mixup, thus, CE loss cooperated with mixup is also compared.

State-of-the-art Methods. The recently proposed representation learning method, namely, OLTR [16] and logit adjustment method, namely, TDE inference [25] are compared. We also compare with the two-stage methods including LDAM-DRW [2] and MisLAS [41], which both achieve satisfactory classification accuracy on the aforementioned long-tailed datasets. For CIFAR-10/100-LT datasets, we make comparison with BBN [42] and contrastive learning [31]. For the large-scale datasets, we compare with the most recently proposed two-stage methods including decoupling [10], Logit adjustment [17] and DisAlign [37]. For a fair comparison, we additionally conduct the comparison experiment with the two-stage strategy which is adding cRT [10] to CE loss + mixup on all datasets.

\subsection{Comparison Results}

Extensive comparative experiments are conducted to illustrate the efficacy of our proposed GCL. The results are presented in Tab. 1 and Tab. 2. We use top-1 accuracy on test sets as the performance metric. For the results that the papers have yet released the official code or relevant hyperparameters, we directly quote their results from the original papers. 
Table 1. Comparison results on CIFAR-10/100-LT. Top-1 accuracy (\%) is reported. The best and the second-best results are shown in underline bold and bold, respectively. *indicates that the results are quoted from the corresponding references. Other results are obtained by re-implementing with the official codes.

\begin{tabular}{|c|c|c|c|c|c|c|}
\hline Dataset & \multicolumn{3}{|c|}{ CIFAR-10-LT } & \multicolumn{3}{|c|}{ CIFAR-100-LT } \\
\hline Backbone Net & \multicolumn{6}{|c|}{ ResNet-32 } \\
\hline imbalance ratio & 200 & 100 & 50 & 200 & 100 & 50 \\
\hline CE loss & 65.68 & 70.70 & 74.81 & 34.84 & 38.43 & 43.9 \\
\hline CE loss + mixup [35] (2018) & 65.84 & 72.96 & 79.48 & 35.84 & 40.01 & 45.16 \\
\hline LDAM-DRW [2] (2019) & 73.52 & 77.03 & 81.03 & 38.91 & 42.04 & 47.62 \\
\hline De-confound-TDE * [25] (2020) & - & 80.60 & 83.60 & - & 44.15 & 50.31 \\
\hline $\mathrm{CE}$ loss + mixup + cRT [10] (2020) & 73.06 & 79.15 & 84.21 & 41.73 & 45.12 & 50.86 \\
\hline $\mathrm{BBN}[42](2020)$ & 73.47 & 79.82 & 81.18 & 37.21 & 42.56 & 47.02 \\
\hline Contrastive learning $*$ [31] (2021) & - & 81.40 & 85.36 & - & 46.72 & 51.87 \\
\hline MisLAS [41] (2021) & 77.31 & 82.06 & 85.16 & 42.33 & 47.50 & 52.62 \\
\hline GCL & 79.03 & $\underline{82.68}$ & $\underline{85.46}$ & 44.88 & 48.71 & 53.55 \\
\hline
\end{tabular}

Table 2. Comparison results on ImageNet-LT, iNaturalist 2018 and Places-LT. Top-1 accuracy (\%) is reported. The best and the second-best results are shown in underline bold and bold, respectively. *indicates that the results are quoted from the corresponding references. Other results are obtained by re-implementing with the official codes.

\begin{tabular}{c|c|c|c}
\hline Dataset & ImageNet-LT & iNaturalist 2018 & Places-LT \\
\hline \hline Backbone Net & ResNet-50 & ResNet-50 & ResNet-152 \\
\hline CE loss & 44.51 & 63.80 & 27.13 \\
CE loss + mixup [35] (2018) & 45.66 & 65.77 & 29.51 \\
\hline LDAM-DRW [2] * (2019) $_{\text {OLTR * [16] (2019) }}^{*}$ (16.80 & 68.00 & - \\
Decoupling [10] (2020) & - & - & 35.9 \\
CE loss + mixup + cRT [10] (2020) & 47.70 & 69.49 & 37.62 \\
Logit adjustment * [17](2021) & 51.68 & 70.16 & 38.51 \\
DisAlign * [37] (2021) & 51.11 & 66.36 & - \\
MisLAS [41] (2021) & $\mathbf{5 2 . 9 1}$ & 70.06 & 39.30 \\
\hline GCL & 52.11 & $\mathbf{7 1 . 5 7}$ & $\mathbf{4 0 . 1 5}$ \\
\hline
\end{tabular}

\subsubsection{Experimental Results on CIFAR-10/100-LT}

The results on CIFAR-10/100-LT datasets are summarized in Tab. 1. We can observe that our proposed GCL outperforms the previous methods by notable margins with all imbalanced ratios. Especially for the largest one, i.e., $\gamma=200$, the proposed approach has obvious improvement. We get $79.03 \%$ and $44.88 \%$ in top- 1 classification accuracy for CIFAR-10-LT and CIFAR-100-LT with $\gamma=200$, which surpasses the second best method, i.e., MisLAS by a significant margin of $1.72 \%$ and $2.55 \%$, respectively.

\subsubsection{Experimental Results on Large-scale Latasets}

The results on three large-scale long-tailed datasets, i.e., ImageNet-LT, iNaturalist 2018, and Place-LT are reported in Tab. 2. Our approach is superior to prior art on all datasets. On ImageNet-LT, our method achieves 54.88\% top-1 accuracy, outperforming DisAlign by a large margin at $1.97 \%$ and MisLAS at $2.77 \%$, respectively. On iNaturalist 2018 , the proposed approach achieves $72.01 \%$ top-
1 accuracy, which outperforms the second-best method by $0.44 \%$. On Place-LT, our method achieves $40.64 \%$ top- 1 classification accuracy, with a performance gain at $0.49 \%$ over MisLAS. Although the performance gain compared with MisLAS on iNaturalist 2018 and Place-LT is not as high as other datasets, our method does not require hyperparameters searching for different datasets, and thus is relatively easy to implement.

\subsection{Model Validation and Analysis}

We conduct a series of ablation studies to further analyze the proposed method.

\subsubsection{The Role of Gaussian Clouded Logit}

In order to obtain additional insight, we use t-SNE projection of the embedding for visualization. Since the loss functions of baseline and MisLAS are both CE loss, moreover, MisLAS performs the second-best in most cases, we visualize $\mathrm{CE}$ loss embedding for comparison. The embeddings are calculated from the samples in CIFAR-10-LT with 


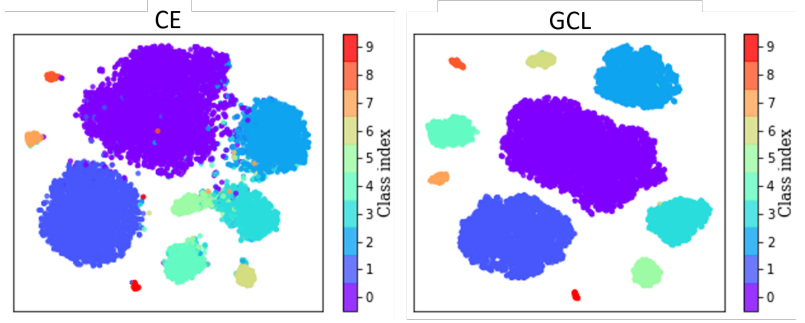

(a) On training set

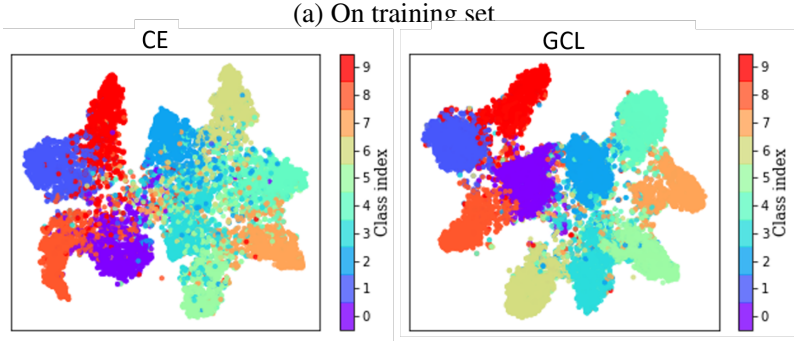

(b) On test set

Figure 4. Visualization of the embedding via t-SNE from CIFAR10-LT with $\gamma=100$. The backbone network is ResNet-32. (Color for the best view.)

Table 3. Ablation experiment of different cloud size adjustment strategies (AS) on CIFAR-10-LT with $\gamma=100$.

\begin{tabular}{ccc}
\hline AS & Expression & $\operatorname{Acc}(\%)$ \\
\hline \hline $\cos$. & $\cos \left(n_{j} / n_{\max } \cdot \pi / 2\right)$ & 79.21 \\
pow. diff. (e:1/3) & $n_{\max }^{1 / 3}-n_{j}^{1 / 3}$ & 80.80 \\
pow. diff. (e:1/4) & $n_{\max }^{1 / 4}-n_{j}^{1 / 4}$ & 82.31 \\
$\log$. diff. & $\log n_{\max }-\log n_{j}$ & $\underline{\mathbf{8 2 . 6 8}}$ \\
\hline
\end{tabular}

$\gamma=100$. Fig. 4 shows the visualization results on the training and test set. From the result of the training set (Fig. 4a), we can see that the embeddings obtained via GCL of different classes are more scattered. Therefore, the GCL embedding of each class is much easier to separate. The results of the test set shown in Fig. 4b verify the efficacy of our proposed approach. The obscure region of CE loss embedding is larger than that of GCL embedding. Good embedding helps improve the model performance. We only re-fine the classifier with the simple cRT without any other complicated technologies, the classification accuracy can be improved a lot.

\subsubsection{Cloud Size Adjustment Strategy}

We explore several different cloud size adjustment strategies (AS), which include cosine form (cos.), power difference (pow. diff.) with different exponents (e:1/3 and e:1/4), and logarithmic difference (log. diff.). For a fair comparison, the sampler and re-training strategy are selected as CBEN and cRT, respectively. Tab. 3 shows the results. We choose the Log. diff. strategy according Tab. 3.
Table 4. Ablation experiment Table 5. Ablation experiment of different re-sampling strategy of different re-training strategies on CIFAR-10-LT with $\gamma=100$. on CIFAR-10-LT with $\gamma=100$.

\begin{tabular}{|c|c|c|c|c|c|}
\hline sam. & RT & $\operatorname{Acc}(\%)$ & sam. & RT & $\operatorname{Acc}(\%)$ \\
\hline IB & cRT & 80.41 & - & w/o RT & 80.52 \\
\hline $\mathrm{CB}$ & cRT & 82.43 & CBEN & LWS & 82.25 \\
\hline EN & cRT & 82.47 & CBEN & $\tau$-nor. & 82.16 \\
\hline CBEN & cRT & 82.68 & CBEN & cRT & 82.68 \\
\hline
\end{tabular}

\subsubsection{Classifier Re-balance Strategies}

We compare different strategies of data re-sampling and the classifier re-training to better analyze our proposed method. The re-sampling strategy (sam.) includes: instance balance (IB) [10], class balance (CB) [10], class balance with effective number (EN) [4] and our proposed class-based effective number (CBEN). For a fair comparison, the re-training strategies for all samplers are cRT. Tab. 4 shows the effectiveness of CBEN. For the selection of classifier re-training strategy, we first train the backbone without any classifier re-training technology. Then we fix the representation and re-balance the classifier with learnable weight scaling (LWS), $\tau$-normalized ( $\tau$-nor.), and cRT, respectively. Tab. 5 presents the top- 1 accuracy of CIFAR-10-LT with $\rho=100$. We can observe that even without any classifier re-training technique, our approach can still beat most state-of-the-arts including two-stage methods. For example, our GCL without classifier re-training suppresses BBN by $0.7 \%$. And cRT outperforms best among the classifier re-training strategies, which improves the top- 1 accuracy by $1.64 \%$. From Tab. 4 and Tab. 5, we can observe that IB+cRT degrades model performance, which indicates that training the classifier with IB leads to classifier overfitting.

\section{Conclusion}

In this paper, we have found that softmax saturation reduces sample validity, which has different effects on head and tail classes. This implies that, from another perspective, softmax saturation can be utilized to automatically adjust the training sample validity of different classes. Subsequently, we have proposed the Gaussian clouded logit (GCL). The tail class logits are set to relatively large cloud sizes to encourage more tail class samples to participate in training as well as leave large margins, which help obtain evenly distributed embedding space. The effectiveness of different classes is varied via GCL. Then, the simple but effective class-based effective number (CBEN) sampling strategy incorporated with Classifier re-training (cRT) for classifier balancing has been proposed, which can further boost the model performance. Extensive experiments on various benchmark datasets have demonstrated that the proposed Gaussian clouded logit obtains superior performance compared to the existing state-of-the-art methods. 


\section{References}

[1] Dong Cao, Xiangyu Zhu, Xingyu Huang, Jianzhu Guo, and Zhen Lei. Domain balancing: Face recognition on longtailed domains. In CVPR, June 2020. 3

[2] Kaidi Cao, Colin Wei, Adrien Gaidon, Nikos Arechiga, and Tengyu Ma. Learning imbalanced datasets with labeldistribution-aware margin loss. In NeurIPS, pages 15671578, 2019. 1, 3, 4, 6, 7

[3] Binghui Chen, Weihong Deng, and Junping Du. Noisy softmax: Improving the generalization ability of denn via postponing the early softmax saturation. In $C V P R$, July 2017. 2, 3

[4] Yin Cui, Menglin Jia, Tsung-Yi Lin, Yang Song, and Serge Belongie. Class-balanced loss based on effective number of samples. In $C V P R$, pages 9268-9277, 2019. 2, 3, 5, 6, 8

[5] Jiankang Deng, Jia Guo, Niannan Xue, and Stefanos Zafeiriou. Arcface: Additive angular margin loss for deep face recognition. In $C V P R$, pages 4690-4699, 2019. 4

[6] Kaiming He, Georgia Gkioxari, Piotr Dollár, and Ross Girshick. Mask r-cnn. In ICCV, pages 2961-2969, 2017. 1

[7] Kaiming He, Xiangyu Zhang, Shaoqing Ren, and Jian Sun. Deep residual learning for image recognition. In $C V P R$, pages 770-778, 2016. 1

[8] Youngkyu Hong, Seungju Han, Kwanghee Choi, Seokjun Seo, Beomsu Kim, and Buru Chang. Disentangling label distribution for long-tailed visual recognition. In $C V P R$, pages 6626-6636, June 2021. 1, 3

[9] Chen Huang, Yining Li, Chen Change Loy, and Xiaoou Tang. Learning deep representation for imbalanced classification. In $C V P R$, June 2016. 3

[10] Bingyi Kang, Saining Xie, Marcus Rohrbach, Zhicheng Yan, Albert Gordo, Jiashi Feng, and Yannis Kalantidis. Decoupling representation and classifier for long-tailed recognition. In ICLR, 2020. 1, 3, 5, 6, 7, 8

[11] Salman H. Khan, Munawar Hayat, Mohammed Bennamoun, Ferdous Ahmed Sohel, and Roberto Togneri. Costsensitive learning of deep feature representations from imbalanced data. IEEE Trans. Neural Networks Learn. Syst., 29(8):3573-3587, 2018. 3

[12] Jang-Hyun Kim, Wonho Choo, Hosan Jeong, and Hyun Oh Song. Co-mixup: Saliency guided joint mixup with supermodular diversity. In ICLR, 2021. 6

[13] Pang Wei Koh and Percy Liang. Understanding black-box predictions via influence functions. In ICML, volume 70 of Proceedings of Machine Learning Research, pages 18851894, 2017. 3

[14] Alex Krizhevsky, Geoffrey Hinton, et al. Learning multiple layers of features from tiny images. Tech Report, 2009. 6

[15] Tsung-Yi Lin, Priya Goyal, Ross B. Girshick, Kaiming He, and Piotr Dollár. Focal loss for dense object detection. IEEE TPAMI, 42(2):318-327, 2020. 2

[16] Ziwei Liu, Zhongqi Miao, Xiaohang Zhan, Jiayun Wang, Boqing Gong, and Stella X. Yu. Large-scale long-tailed recognition in an open world. In $C V P R$, pages 2537-2546, 2019. 6, 7

[17] Aditya Krishna Menon, Sadeep Jayasumana, Ankit Singh Rawat, Himanshu Jain, Andreas Veit, and Sanjiv Kumar.
Long-tail learning via logit adjustment. In ICLR, 2021. 1, $3,6,7$

[18] Tianyu Pang, Kun Xu, and Jun Zhu. Mixup inference: Better exploiting mixup to defend adversarial attacks. In ICLR, 2020. 6

[19] Adam Paszke, Sam Gross, Francisco Massa, Adam Lerer, James Bradbury, Gregory Chanan, Trevor Killeen, Zeming Lin, Natalia Gimelshein, Luca Antiga, et al. Pytorch: An imperative style, high-performance deep learning library. In NeurIPS, pages 8024-8035, Vancouver, BC, Canada, Dec. 2019. 6

[20] Mengye Ren, Wenyuan Zeng, Bin Yang, and Raquel Urtasun. Learning to reweight examples for robust deep learning. In $I C M L$, volume 80, pages 4331-4340, 2018. 2

[21] Shaoqing Ren, Kaiming He, Ross Girshick, and Jian Sun. Faster r-cnn: towards real-time object detection with region proposal networks. IEEE TPAMI, 39(6):1137-1149, 2016. 1

[22] Olga Russakovsky, Jia Deng, Hao Su, Jonathan Krause, Sanjeev Satheesh, Sean Ma, Zhiheng Huang, Andrej Karpathy, Aditya Khosla, Michael Bernstein, Alexander C. Berg, and $\mathrm{Li}$ Fei-Fei. Imagenet large scale visual recognition challenge. IJCV, 115(3):211-252, 2015. 6

[23] Jingru Tan, Changbao Wang, Buyu Li, Quanquan Li, Wanli Ouyang, Changqing Yin, and Junjie Yan. Equalization loss for long-tailed object recognition. In CVPR, pages 1165911668. Computer Vision Foundation / IEEE, 2020. 3

[24] Kaihua Tang, Jianqiang Huang, and Hanwang Zhang. Longtailed classification by keeping the good and removing the bad momentum causal effect. In NeurIPS, 2020. 3

[25] Kaihua Tang, Jianqiang Huang, and Hanwang Zhang. Longtailed classification by keeping the good and removing the bad momentum causal effect. In NeurIPS, 2020. 6, 7

[26] Laurens Van der Maaten and Geoffrey Hinton. Visualizing data using t-sne. Journal of machine learning research, 9(11), 2008. 2

[27] Grant Van Horn, Oisin Mac Aodha, Yang Song, Yin Cui, Chen Sun, Alex Shepard, Hartwig Adam, Pietro Perona, and Serge J. Belongie. The inaturalist species classification and detection dataset. In CVPR, pages 8769-8778, 2018. 6

[28] Vikas Verma, Alex Lamb, Christopher Beckham, Amir Najafi, Ioannis Mitliagkas, David Lopez-Paz, and Yoshua Bengio. Manifold mixup: Better representations by interpolating hidden states. In ICML, pages 6438-6447. PMLR, 2019. 3

[29] Feng Wang, Xiang Xiang, Jian Cheng, and Alan Loddon Yuille. Normface: $\mathrm{L}_{2}$ hypersphere embedding for face verification. In ACM MM, pages 1041-1049, 2017. 1, 4

[30] Hao Wang, Yitong Wang, Zheng Zhou, Xing Ji, Dihong Gong, Jingchao Zhou, Zhifeng Li, and Wei Liu. Cosface: Large margin cosine loss for deep face recognition. In $C V P R$, pages 5265-5274, 2018. 4

[31] Peng Wang, Kai Han, Xiu-Shen Wei, Lei Zhang, and Lei Wang. Contrastive learning based hybrid networks for longtailed image classification. In CVPR, pages 943-952, 2021. 3, 6, 7

[32] Tao Wang, Yu Li, Bingyi Kang, Junnan Li, Junhao Liew, Sheng Tang, Steven Hoi, and Jiashi Feng. The devil is in classification: A simple framework for long-tail instance 
segmentation. In ECCV, pages 728-744. Springer, 2020. 1, 3

[33] Tao Wang, Yu Li, Bingyi Kang, Junnan Li, Jun Hao Liew, Sheng Tang, Steven C. H. Hoi, and Jiashi Feng. The devil is in classification: A simple framework for long-tail instance segmentation. In $E C C V$, volume 12359, pages 728-744, 2020. 5

[34] Xin Wang, Thomas E. Huang, Joseph Gonzalez, Darrell Trevor, and Fisher Yu. Frustratingly simple few-shot object detection. In ICML, volume 119, pages 9919-9928, 2020. 3

[35] Hongyi Zhang, Moustapha Cisse, Yann N Dauphin, and David Lopez-Paz. mixup: Beyond empirical risk minimization. ICLR, 2018. 6, 7

[36] Linjun Zhang, Zhun Deng, Kenji Kawaguchi, Amirata Ghorbani, and James Zou. How does mixup help with robustness and generalization? In ICLR, 2021. 6

[37] Songyang Zhang, Zeming Li, Shipeng Yan, Xuming He, and Jian Sun. Distribution alignment: A unified framework for long-tail visual recognition. In CVPR, pages 2361-2370, 2021. 1, 3, 6, 7

[38] Wanping Zhang, Yongru Chen, Wenming Yang, Guijin Wang, Jing-Hao Xue, and Qingmin Liao. Class-variant margin normalized softmax loss for deep face recognition. IEEE Trans. Neural Networks Learn. Syst., 32(10):47424747, 2021. 2, 4

[39] Yifan Zhang, Bingyi Kang, Bryan Hooi, Shuicheng Yan, and Jiashi Feng. Deep long-tailed learning: A survey. arXiv preprint arXiv:2110.04596, 2021. 1

[40] Yongshun Zhang, Xiu-Shen Wei, Boyan Zhou, and Jianxin Wu. Bag of tricks for long-tailed visual recognition with deep convolutional neural networks. In $A A A I$, pages $3447-$ 3455, 2021. 6

[41] Zhisheng Zhong, Jiequan Cui, Shu Liu, and Jiaya Jia. Improving calibration for long-tailed recognition. In $C V P R$, pages 16489-16498, 2021. 3, 6, 7

[42] Boyan Zhou, Quan Cui, Xiu-Shen Wei, and Zhao-Min Chen. BBN: Bilateral-branch network with cumulative learning for long-tailed visual recognition. In CVPR, pages 9719-9728, 2020. 1, 3, 6, 7

[43] Bolei Zhou, Agata Lapedriza, Aditya Khosla, Aude Oliva, and Antonio Torralba. Places: A 10 million image database for scene recognition. IEEE TPAMI, 40(6):1452-1464, 2017. 6 